\title{
Fibroblasts endocytose and degrade transthyretin aggregates in transthyretin-related amyloidosis
}

\author{
Yohei Misumi ${ }^{1,2}$, Yukio Ando², Nádia P Gonçalves ${ }^{1,3}$ and Maria J Saraiva ${ }^{1,3}$
}

Transthyretin (TTR)-related amyloidosis is a fatal disorder characterized by systemic extracellular deposition of TTR amyloid fibrils. Mutations in the TTR gene cause an autosomal dominant form of the disease-familial amyloidotic polyneuropathy (FAP). Wild-type (WT) TTR can also form amyloid fibrils in elderly patients with senile systemic amyloidosis. Regression of amyloid deposits in FAP patients who undergo liver transplantation to remove the main source of mutant TTR suggests the existence of mechanisms for the clearance of TTR deposits from the extracellular matrix $(E C M)$, but the precise mechanisms are largely unknown. Because fibroblasts are abundant, playing a central role in the maintenance of the ECM and because the skin is one of the major sites of soluble TTR catabolism, in the present study, we analyzed their role in clearance of TTR aggregates. In vitro studies with a fibroblast cell line revealed that fibroblasts endocytosed and degraded aggregated TTR. Subcutaneous injection of soluble and aggregated TTR into WT mice showed internalization and clearance over time by both fibroblasts and macrophages. Immunohistochemical studies of skin biopsies from V30M patients, asymptomatic carriers, recipients of domino FAP livers as well as transgenic mice for human V30M showed intracellular TTR immunoreactivity in fibroblasts and macrophages that increased with clinical status and with age in transgenic mice. Overall, the present in vitro and in vivo data show that fibroblasts endocytose and degrade TTR aggregates. The function or dysfunction of TTR clearance by fibroblasts may have important implications for the development, progression, and regression of TTR deposition in the ECM.

Laboratory Investigation (2013) 93, 911-920; doi:10.1038/labinvest.2013.83; published online 1 July 2013

KEYWORDS: amyloidosis; endocytosis; extracellular matrix; familial amyloidotic polyneuropathy; fibroblasts; transthyretin; TTR degradation

Transthyretin (TTR)-related amyloidoses are fatal systemic amyloidoses associated with systemic deposition of TTR extracellular amyloid fibrils. TTR-related familial amyloidotic polyneuropathy (FAP) is an autosomal dominant disease related to mutant TTR deposition in various organs and systems, including the peripheral nervous system, eyes, heart, gastrointestinal tract, kidneys, and skin. As of 2012, more than 120 TTR mutations have been discovered, most of which lead to FAP development (http://www.fapwtr.org/ ram_fap.htm); TTR V30M is the most common diseasecausing mutation and occurs in large foci of patients throughout the world. ${ }^{1}$ Senile systemic amyloidosis (SSA) is an age-related nonhereditary systemic amyloidosis and mainly affects cardiac functions in elderly people where wild-type (WT) TTR forms amyloid in different tissues. ${ }^{2}$

TTR is primarily synthesized by the liver, retina, and choroid plexus of brain, circulates as a soluble tetramer form in blood, cerebrospinal fluid, and aqueous humor, and transports thyroxine and retinol-binding protein. TTR dissociation into monomers is a determining step for partial misfolding, oligomer formation, and fibrillogenesis in tissues. The rate of TTR tetramer dissociation is increased by amyloidogenic mutations. ${ }^{3}$ The pathogenesis of TTR-related amyloidosis, however, cannot be fully explained by instability of the tetrameric structure that results from mutations because of the following: (1) the penetrance, age at onset, clinical symptoms, and prognosis differ depending on race and pedigree, even for the same mutant TTR; ${ }^{4}(2)$ WT TTR also forms amyloid fibrils and causes SSA in elderly people, especially men; ${ }^{2}$ and (3) pathological studies reveal organ and tissue tropism of TTR amyloid. ${ }^{5}$ These data indicate the presence of complex mechanisms of amyloid fibril formation and clearance in vivo, mechanisms that may be affected by unknown genetic and environmental factors, aging, and sex differences.

${ }^{1}$ Molecular Neurobiology, IBMC — Instituto de Biologia Molecular e Celular, Porto, Portugal; ${ }^{2}$ Department of Neurology, Graduate School of Medical Sciences, Kumamoto University, Kumamoto, Japan and ${ }^{3}$ Instituto de Ciências Biomédicas Abel Salazar, ICBAS, University of Porto, Porto, Portugal Correspondence: Professor MJ Saraiva, PhD, Molecular Neurobiology, IBMC - Instituto de Biologia Molecular e Celular, 823 Rua do Campo Alegre, Porto 4150-180, Portugal. E-mail: mjsaraiv@ibmc.up.pt 
Previous histopathological observations on V30M individuals showed extracellular oligomeric non-fibrillar Congo red-negative TTR forms in tissues of FAP patients, asymptomatic carriers, recipients of livers transplanted from FAP patients and transgenic mouse models expressing amyloidogenic TTR V30M. ${ }^{6-8}$ Interestingly, pathological and biochemical findings of FAP patients who underwent liver transplantation to remove the main source of mutant TTR showed regression of amyloid deposits in aspirated abdominal fat tissues and gastrointestinal mucosa. ${ }^{9}$ Although cardiac amyloid deposition reportedly sometimes progressed after liver transplantation, proteomics analyses revealed that amyloid fibrils in these patients were derived primarily from WT TTR, which the normal liver graft must have produced. ${ }^{10}$ These findings suggest the presence of a dynamic balance between the formation and degradation of TTR amyloid in the extracellular matrix (ECM).

A kinetic study of serum WT TTR showed that TTR is degraded in the liver, muscle, skin, and kidney. ${ }^{11}$ Different tetrameric stabilities of WT and mutant TTRs cause different clearance patterns from plasma. ${ }^{12}$ Previous in vitro studies showed that WT and mutant TTR are internalized by different types of cells, including hepatocytes, mouse embryonic fibroblasts, yolk sac cells, and sensory neurons. ${ }^{13-15}$ Whether these cells could endocytose and degrade TTR aggregates in vivo needs investigation.

The present report addresses this question in a fibroblast cell line and an experimental mouse model after subcutaneous injection of TTR aggregates. Furthermore, we inspected the presence of TTR in fibroblasts in skin biopsies of V30M FAP patients, asymptomatic carriers, recipients of domino FAP transplants, and also in V30M transgenic mice at various ages.

\section{MATERIALS AND METHODS Tissue Samples}

Archival skin biopsy samples obtained from FAP V30M patients $(n=4)$, asymptomatic carriers $(n=4)$, domino liver transplant recipients $(n=15)$, and normal control subjects $(n=4)$ were previously characterized for TTR deposition after informed consent. ${ }^{7}$ Autopsy heart tissues from six patients with FAP V30M and six non-FAP patients who had no heart disease were obtained at Kumamoto University Hospital. The Ethical Committee of Kumamoto University gave approval for the study.

Skin specimens from transgenic mice expressing human TTR V30M in a TTR null background at the ages of $3(n=5)$, $6(n=5)$, and $15(n=5)$ months were available at the laboratory. ${ }^{16}$

\section{Subcutaneous (s.c.) Injection of TTR into Mice}

Mice were housed in pathogen-free conditions; all animal experiments were performed according to the European Communities Council Directive. To investigate the function of fibroblasts in the degradation of TTR in vivo, we injected 6-month-old SV129 WT mice s.c. in three different spots in the back using $29 \mathrm{G}$ syringes with preparations containing $0.05 \mathrm{mg}$ (in $200 \mu \mathrm{l}$ volume) of either soluble or aggregated WT TTR and V30M TTR ( $\mathrm{n}=3$ /group). Skin samples were collected (including approximately $2 \mathrm{~mm}$ of surrounding tissue from the center of injected site) 3,8 , and $24 \mathrm{~h}$ after injection, subsequently fixed in formalin, and embedded in paraffin. Three-micrometer slices were cut and evaluated by immunohistochemistry.

\section{Preparation of Soluble TTR and Aggregated TTR}

Recombinant WT and V30M TTRs were produced in an Escherichia coli expression system. Protein isolation and purification were performed as previously described. ${ }^{17}$ Aggregated TTR forms were generated by incubating the protein $(0.25 \mathrm{mg} / \mathrm{ml})$, with stirring, at room temperature for 7 days. ${ }^{18}$ Aggregated TTR was diluted 1:9 (v/v) with $30 \mu \mathrm{M}$ thioflavin $\mathrm{T}$ in Glycine Buffer ( $\mathrm{pH}$ 9.0), and the oligomeric preparations were tested by thioflavin $\mathrm{T}$ spectrofluorometric assays (excitation at $440 \mathrm{~nm}$ ). Electron microscopy was also performed to check the aggregates formation. For fluorescent TTR, the protein was labelled with the fluorescent dye Alexa Fluor 488 (Invitrogen, Carlsbad, CA, USA) according to the manufacturer's instructions.

\section{Cell Culture}

The human fibroblast cell line NIH3T3 was cultured in Dulbecco's Modified Eagle Medium (DMEM) supplemented with $10 \%$ fetal bovine serum (FBS) and penicillin-streptomycin $(0.1 \mathrm{mg} / \mathrm{ml})$ at $37^{\circ} \mathrm{C}$ in a $5 \% \mathrm{CO}_{2}$ humidified atmosphere. Multi-spot glass slides (Greiner Bio-One, Frickenhausen, Germany) and 12-well plates (Orange Scientific, Braine-l'Alleud, Belgium) were coated with $250 \mathrm{ng}$ of aggregated TTR prepared as described above, air-dried, and incubated with NIH3T3 cells $\left(1 \times 10^{4}\right.$ cells $\left./ \mathrm{ml}\right)$ in DMEM for up to $48 \mathrm{~h}$. For immunocytochemistry, cells on glass slides were washed twice with phosphate-buffered saline (PBS) and fixed for 30 min with $2 \%$ paraformaldehyde before incubation with selected antibodies; for immunoblotting assays, the 12-well coated plates were treated with $20 \mathrm{mM}$ HEPES-NaOH ( $\mathrm{pH}$ 7.4) containing $1 \%$ Triton X-100 before application into filters. In another experiments, fibroblasts were incubated with unlabelled TTR aggregates $(1 \mu \mathrm{g} / \mathrm{ml})$ in 24-well plates (Orange Scientific), and supernatants were collected at $0,3,8,24$, and $48 \mathrm{~h}$ (three wells per time point) and measured for total and aggregated TTR content (see below). For live cell culture studies, cells were incubated in chamber slides (Ibidi, Munich, Germany) with either synthetic soluble Alexa-TTR or Alexa TTR aggregates for $24 \mathrm{~h}$ and then incubated with red fluorescent LysoTracker (Invitrogen Molecular Probes, Eugene, OR, USA) for $60 \mathrm{~min}$, after which images of the fluorescence were acquired with a confocal microscope (Leica TCS SP2 AOBS; Leica Microsystems, Heidelberg, Germany). Experiments were performed in duplicate and repeated three times. 


\section{Dot-Blot and Filter Assays}

Amounts of total TTR and TTR aggregates were analyzed by dot-blot and filter assays, respectively, as previously described. ${ }^{19}$ For the dot-blot assay, cell culture medium or lysate was applied to a nitrocellulose membrane and dried. For the filter assay, to separate soluble proteins from aggregates, samples were applied to a $0.2-\mu \mathrm{m}$-pore cellulose acetate membrane filter (Schleicher and Schuell, Dassel, Germany), by using a filtration manifold system (Gibco BRL, Gaithersburg, MD, USA). The membrane/filter were blocked with $5 \%(\mathrm{w} / \mathrm{v})$ skim milk in PBS for $1 \mathrm{~h}$, incubated with a rabbit anti-human TTR antibody (1:500; Dako, Glostrup, Denmark) for $1 \mathrm{~h}$, and then incubated with a horseradish peroxidase-conjugated anti-rabbit IgG (1:500). TTR signals were detected with the ECL Kit (Enhanced Chemiluminescence; GE Healthcare, Buckinghamshire, UK) and were quantified by using the public domain NIH ImageJ program.

\section{Immunohistochemistry and Immunofluorescence}

For immunohistochemistry, tissue sections were treated with $3 \%$ hydrogen peroxide in methanol for $30 \mathrm{~min}$. Slides were incubated in $4 \%$ FBS and $1 \%$ bovine serum albumin in PBS at room temperature. After overnight incubation with antihuman TTR antibody $\left(1: 1000\right.$; Dako) at $4{ }^{\circ} \mathrm{C}$, reactivity was visualized via the biotin-extravidin-peroxidase kit (SigmaAldrich, St Louis, MO, USA). Images were acquired from 10 visual fields per sample at an original magnification of $\times 40$. Calculation of the percentage of TTR-immunopositive area was performed by using NIH ImageJ, as previously described. $^{20}$

For immunofluorescence, primary antibodies were: sheep anti-human TTR (1:500; Abcam, Cambridge, UK), goat anti-S100A4 (1:50; Acris Antibodies GmbH, Herford, Germany), goat anti-Ibal (1:50; Abcam), and rabbit antiLamp2 (1:100; Sigma-Aldrich), which were incubated overnight at $4{ }^{\circ} \mathrm{C}$ in blocking buffer. Secondary antibodies were Alexa Fluor 568 anti-rabbit, Alexa Fluor 568 anti-goat, and Alexa Fluor 488 anti-sheep (1:1000; Invitrogen Molecular Probes), with incubation for $1 \mathrm{~h}$ at room temperature. Slides were mounted with Vectashield (Vector Laboratories, Burlingame, CA, USA) and visualized by confocal microscopy (Leica TCS SP2 AOBS). TTR-immunopositive fibroblasts were detected by merged images with anti-TTR antibody (green) and fibroblast marker (S100A4; red). The number of TTR-immunopositive cells in each group was calculated as an average of 10 visual fields $(200 \mu \mathrm{m}$ $\times 200 \mu \mathrm{m})$ per sample $(1$ specimen/sample $)$ at an original magnification of $\times 40$.

\section{Congo Red Staining}

Paraffin-embedded sections and paraformaldehyde-fixed cells were stained with phenol Congo red solution and were viewed by bright field or polarized light microscope.

\section{Statistics}

All data are expressed as means \pm SD. Controls and treated groups were compared by ANOVA followed by the Tukey post hoc test. Accepted level of significance was $P<0.05$.

\section{RESULTS}

Fibroblasts Endocytose and Degrade TTR Aggregates In Vitro

We performed different types of 'in vitro' studies in NIH3T3 fibroblasts incubated with aggregates preparations formed from WT-TTR. This preparation contains both soluble and oligomeric TTR, with amyloid-like properties as assessed by thioflavin $\mathrm{T}$ (Figure 1a) filter immunoassay (Figure 1b), and electron microscopy (Figure 1c); immunofluorescence analysis with anti-TTR on aggregate-coated glass surfaces (coverslips) showed an heterogeneous preparation of different size aggregates, which is drastically reduced upon addition and incubation with fibroblasts (Figures $1 \mathrm{~d}$ and e); in some instances, aggregates are observed in close contact with the plasma membrane.

The proportion of soluble and aggregated forms was quantified in coated wells in the absence/presence of fibroblasts; when fibroblasts were added, the intra and extracellular total levels of TTR (soluble and aggregated) were significantly reduced (Figure 1f); similarly, levels of aggregated TTR, as assessed by filter assays were also reduced, as compared with the control situation without cells. All together, the data suggested that not only soluble but also aggregated TTR had been degraded. Finally, we found that the levels of total and aggregated TTR decreased in conditioned media over time upon incubation with fibroblasts (Figure 1h).

To investigate internalization of TTR aggregates, we incubated fluorescently labeled soluble and aggregated TTR with fibroblasts and used live cell imaging by confocal microscopy. Consecutive cross-sectional images revealed that indeed fibroblasts internalized small TTR aggregates (Figures 2a and c) observed intracellularly as punctuate material. By contrast, cells cultured with soluble TTR did not display TTR signal (Figure 2b), probably because of inefficient internalization and/or insufficient weak signal. Further incubation with LysoTracker demonstrated co-localization of TTR aggregated material within lysosomes (Figure 2d). Intracellular amyloid formation was not observed in Congo red staining (Supplementary Figure S1a).

\section{Fibroblasts Endocytose and Degrade Soluble and Aggregated TTR in Mouse Skin}

To investigate internalization and degradation 'in vivo', we injected mouse skin with WT and V30M with both soluble and aggregated preparations, killed animals $8 \mathrm{~h}$ later, and searched for TTR immunoreactivity in skin sections. TTR immunoreactivity was observed within a perimeter of approximately $14 \mathrm{~mm}^{2}$ and localized in the dermis and subcutaneous layers but not in the epidermis and muscle 

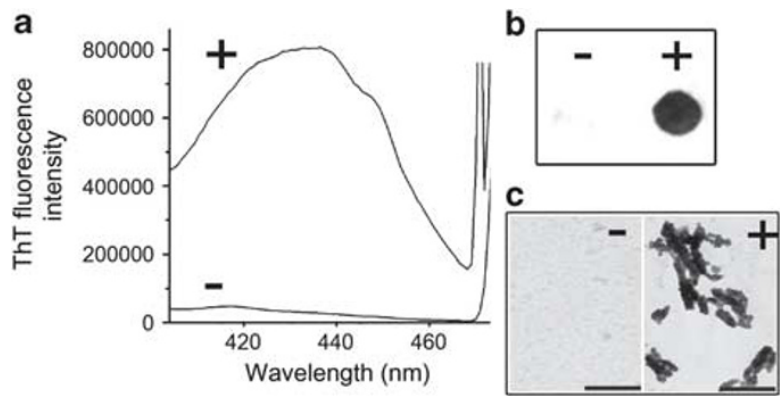

d

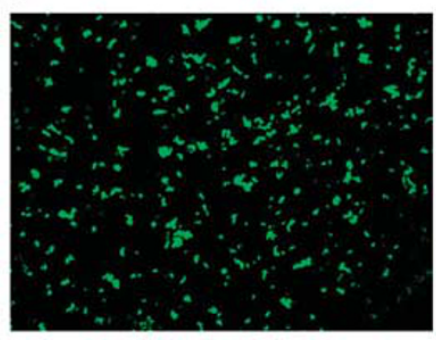

e

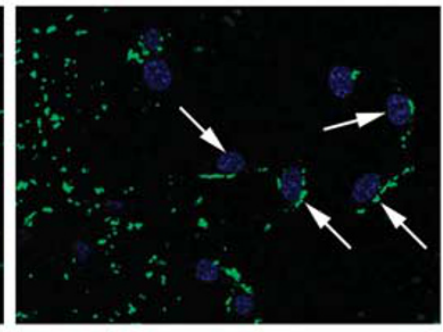

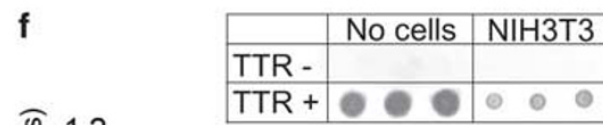
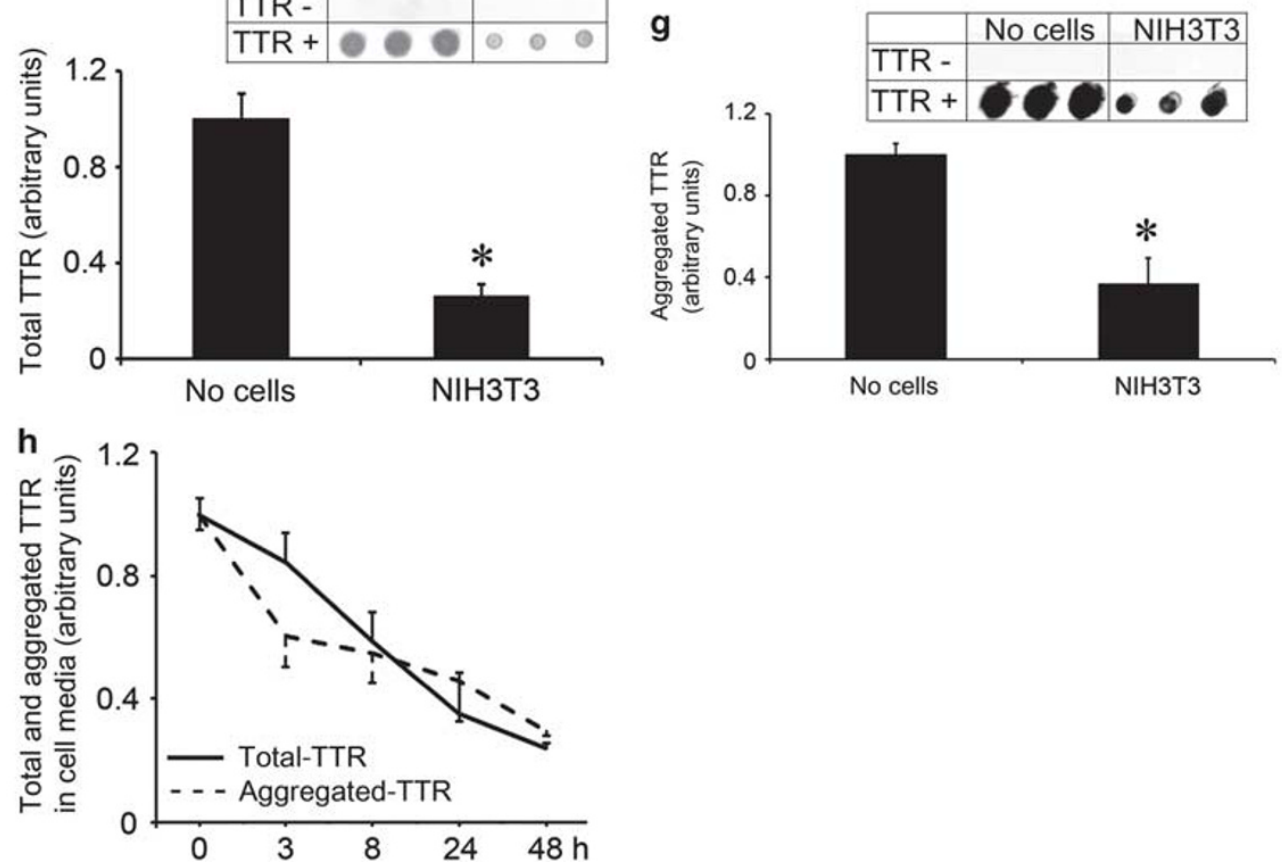

Figure 1 NIH3T3 fibroblasts removed TTR aggregates in vitro. (a) Thioflavin T (ThT) fluorescence spectra of TTR preparations before $(-)$ and after $(+)$ oligomer formation and respective immunoblotting image obtained via (b) the filter assay and (c) electron microscopy. Bar $=100 \mathrm{~nm}$. (d, e) Immunofluorescence images of TTR aggregates (green) on the glass surface after incubation without cells (d) and with NIH3T3 cells (e) Nuclei stained blue with DAPI. Magnification $\times 400$. (f) Quantification of total (soluble and aggregated) TTR on the glass surface as obtained by a dot-blot assay after incubation with and without NIH3T3 cells for $48 \mathrm{~h}$. (g) Quantification of aggregated TTR on the glass surface as obtained by a filter assay after incubation with and without NIH3T3 cells for $48 \mathrm{~h}$. (f, $\mathbf{g}){ }^{*} P<0.01$. (h) Quantification over time of total and aggregated TTR in cell culture media as obtained by dot-blot and filter assays, respectively.

(Figure 3a), in the interstitium, specially around blood vessels and sebaceous glands, and intracellularly in fibroblasts and macrophages (Figure 3a) as confirmed with consecutive crosssectional confocal microscopic images with fibroblast and macrophage markers, respectively (Figures $3 \mathrm{~b}$ and c). Furthermore confocal microscopic images with a lysosomal marker showed that intracellular TTR signals partially co-localized with lysosome (Figure 3d). Other types of cells, including myocytes, epithelial cells, and endothelial cells, showed no intracellular TTR immunoreactivity. No differences in TTR tissue distribution were noticed among tissues from animals injected with WT or V30M either in soluble or aggregated form.

To determine changes in levels of intracellular and extracellular TTR over time, we collected skin samples at 3,8 , and $24 \mathrm{~h}$ after injection and semiquantified total TTR levels and number of immunoreactive fibroblasts. Total TTR, particularly extracellular TTR, constituted most of the TTR found decreased to residual levels at $8 \mathrm{~h}$ and was not detected at $24 \mathrm{~h}$ (Figures $3 \mathrm{f}-\mathrm{i}$ ). The number of TTR-immunopositive fibroblasts, however, increased between 3 and $8 \mathrm{~h}$ and decreased thereafter (Figures $3 \mathrm{f}-\mathrm{h}$ and $\mathrm{j}$ ). Similar decay and/or number of immunoreactive fibroblast patterns were found in the four groups of injected mice (with soluble and aggregated WT and V30M TTR). Intracellular amyloid formation was not detected with Congo red staining (Supplementary Figure S1b).

\section{TTR Co-Localized with Fibroblasts in Transgenic Mice Expressing Human V30M TTR}

In another study, we analyzed skin from 15-month-old transgenic mice expressing human TTR V30M in a TTR null 

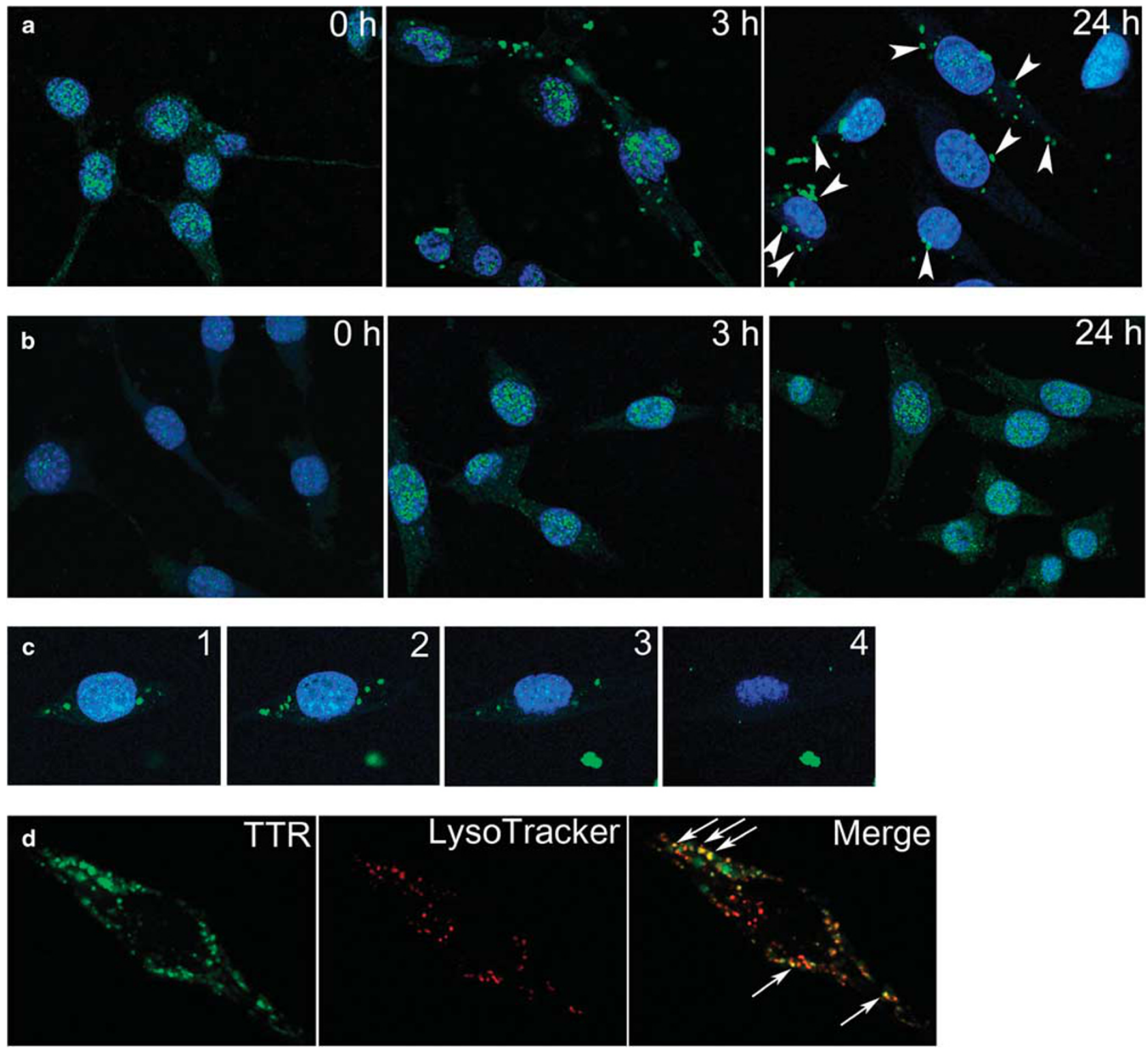

Figure 2 Internalization of TTR aggregates in vitro by NIH3T3 fibroblasts. Fluorescence images of NIH3T3 cells incubated with (a) TTR aggregates (green) or (b) soluble TTR in cell culture media at 0,3 , and $24 \mathrm{~h}$. Arrow heads indicate TTR aggregates close contact with cell membranes. Nuclei stained blue with DAPI. Magnification $\times 400$. (c) Consecutive cross-sectional (A-D) images of NIH3T3 cells after incubation with aggregated fluorescent TTR for $24 \mathrm{~h}$. Magnification $\times 400$. (d) Image of live NIH3T3 cells incubated with fluorescent TTR aggregates (green) and LysoTracker (red). Arrows indicate co-localization of TTR aggregates in lysosomes. Magnification $\times 400$.

background; this strain shows deposition of non-fibrillar TTR after 3 months of age, especially in the gastrointestinal tract and skin; small amounts of TTR amyloid fibrils are detected in the gastrointestinal tract after 9 months of age. ${ }^{16}$ Immunohistochemistry and immunofluorescence with anti-TTR antibody and a fibroblast and/or macrophage marker demonstrated TTR in some fibroblasts and macrophages in the skin, besides the typical extracellular TTR deposits (Figures $4 \mathrm{a}-\mathrm{c}$ ), and shared a similar histology with specimens from WT mice that received s.c. TTR injections as described above; immunopositive tissues from the gastrointestinal tract also disclosed TTR-positive fibroblasts and macrophages (data not shown).
We further investigated TTR deposition at different ages in this mouse model. Extracellular TTR deposition increased in skin with advancing age, as in previous reports. ${ }^{21}$ Concerning intracellular TTR, the number of TTRimmunopositive fibroblasts significantly increased between 3 and 6 months and remained unchanged at 15 months (Figures $4 \mathrm{~d}-\mathrm{g}$ ).

TTR Co- Localized with a Fibroblast Marker in Skin and Heart Specimens from FAP Patients

Finally, to determine whether fibroblasts in human tissues internalize TTR, we performed TTR immunohistochemistry 
a

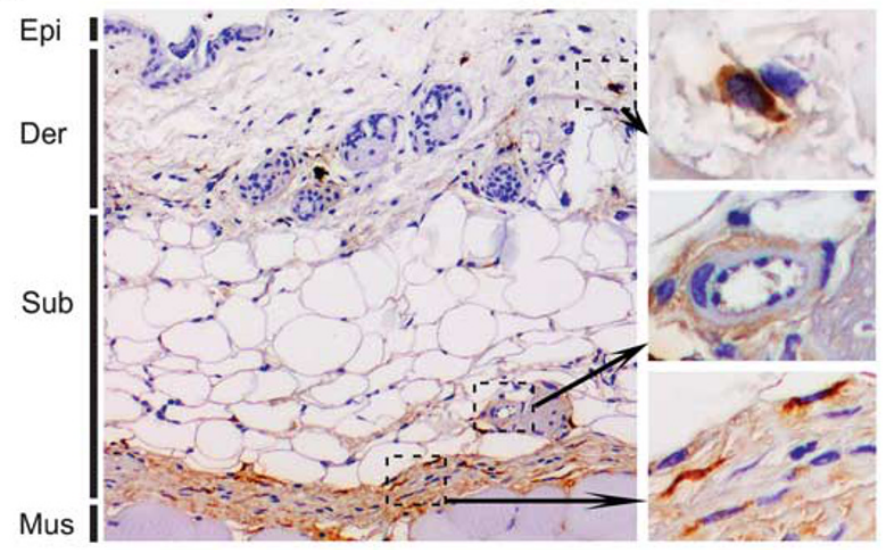

b

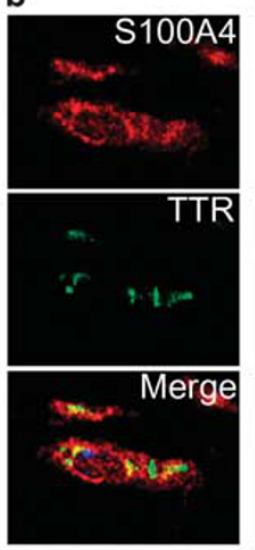

C

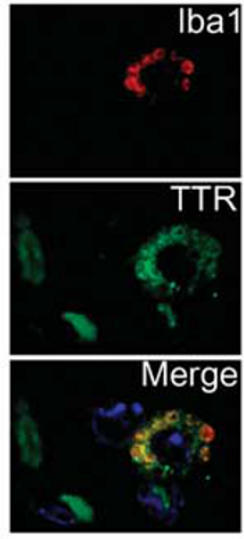

d

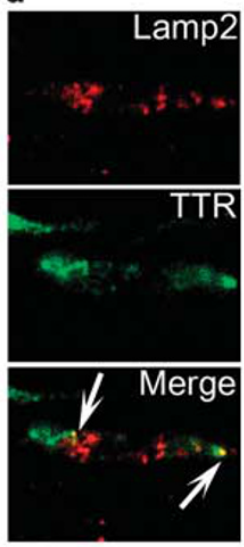

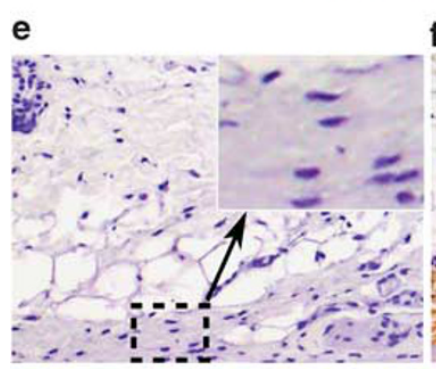

f

g

h
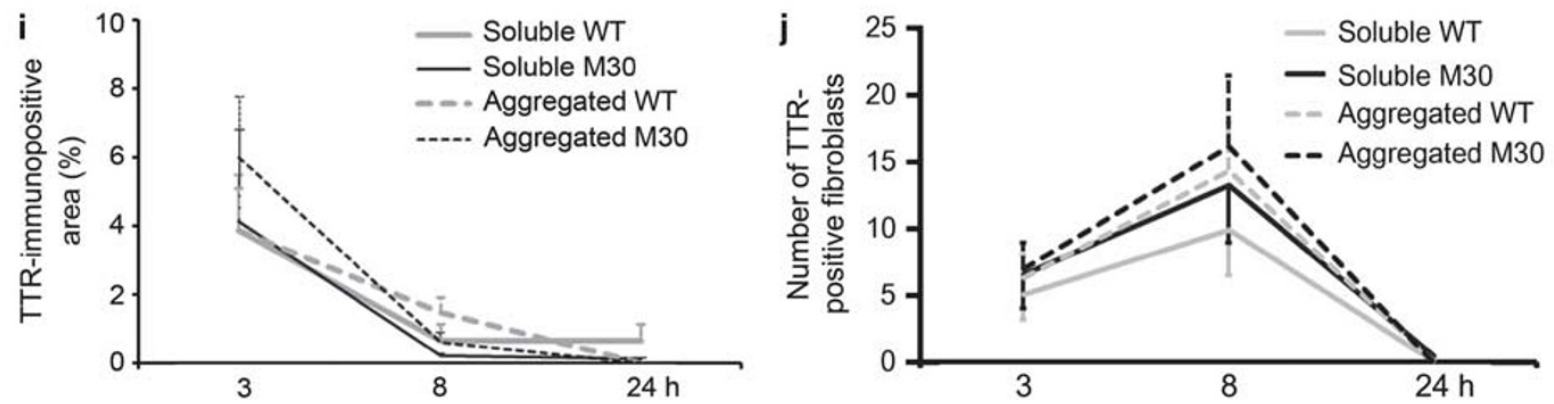

Figure 3 Endocytosis by fibroblasts of TTR injected into mouse skin. (a) TTR immunostaining of a skin sample taken at $8 \mathrm{~h}$ after V30M TTR aggregates injection. Different skin areas are indicated in the left. Magnification $\times 200$. (b) Confocal microscopy image with anti-TTR antibody (green), fibroblast marker (S100A4; red), (c) macrophage marker (lba1; red), and (d) lysosome marker (Lamp2; red). Nuclei stained blue with DAPI. Arrows indicate colocalization of TTR aggregates in lysosomes. (e-h) TTR immunostaining of skin samples taken at (e) $3 \mathrm{~h}$ after PBS injection and $3 \mathrm{~h}(\mathbf{f}), 8 \mathrm{~h}(\mathbf{g})$, and $24 \mathrm{~h}$ (h) after injection of V30M TTR aggregates. Magnification $\times 400$. (i, j) Semiquantification of (i) extracellular TTR-immunopositive areas and (j) the number of TTR-immunopositive fibroblasts after injection of different TTR preparation, both soluble and aggregated TTR derived from WT and V30M mutant. Insets show boxed areas at greater magnification.

and confocal microscopy with the fibroblast marker in skin biopsy samples and autopsy heart tissues obtained from FAP V30M patients containing amyloid deposits, asymptomatic carriers with non fibrillar TTR deposition, domino liver transplant recipients (with non-fibrillar and amyloid deposition), and controls (Figure 5). Except for control skin, all the groups analyzed had TTR-immunoreactive fibroblasts (Figures $5 \mathrm{a}-\mathrm{c}$ ), as confirmed by confocal microscopy with the fibroblast marker (Figure 5d). The proportion of intracellular TTR immunoreactivity was not much, because TTR aggregates were mainly deposited in the extracellular space in tissues from patients. The proportion of fibroblasts with intracellular TTR varied among the analyzed groups as summarized in Table 1; thus, three out of the four skin biopsies from FAP patients presented TTR in fibroblasts, whereas only one out of four asymptomatic carrier samples were positive. As for FAP domino liver transplant recipients, we analyzed skin biopsies post transplant collected after 1-4 years, which were both Congo red and TTR negative and collected after 4 years of transplant; the latter group was positive for TTR and included samples with and without amyloid deposition. Two out five samples that were positive for TTR but negative for Congo red birefringency (CR-TTR + ) showed TTR-immunoreactive fibroblasts, whereas this proportion increased to four out of five in skin biopsies positive for amyloid ( $\mathrm{CR}+\mathrm{TTR}+)$. The levels of TTR-immunoreactive fibroblasts increased significantly in FAP patients as presented in Figure $5 \mathrm{e}$. 
a

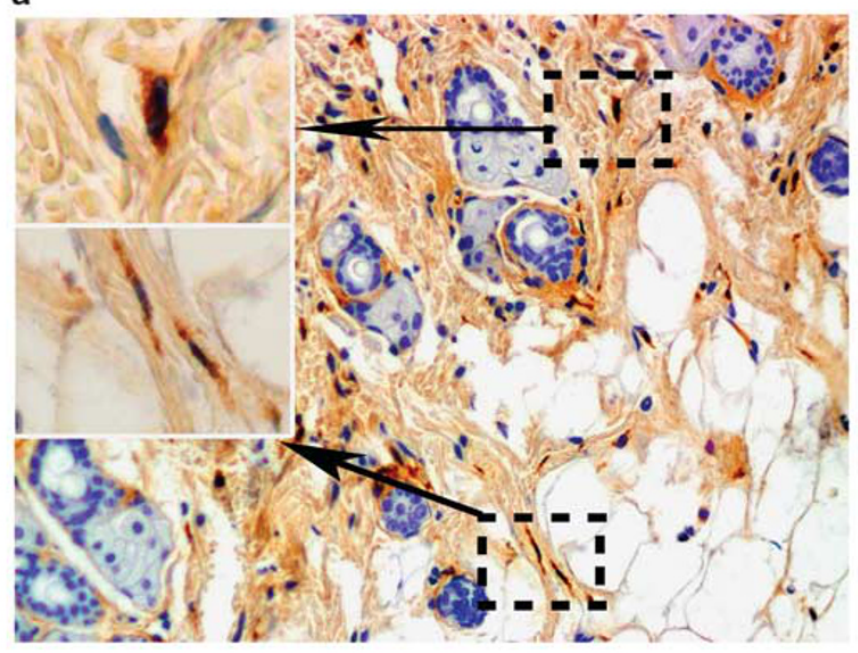

b

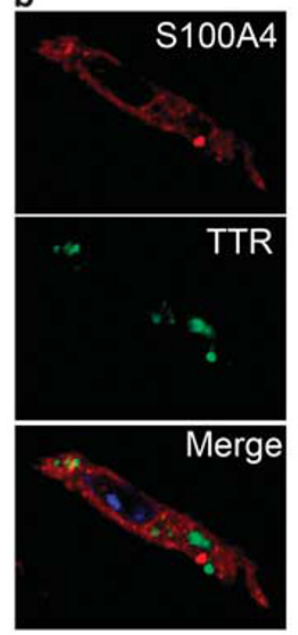

c

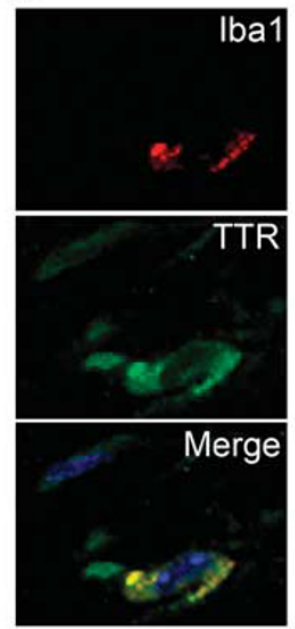

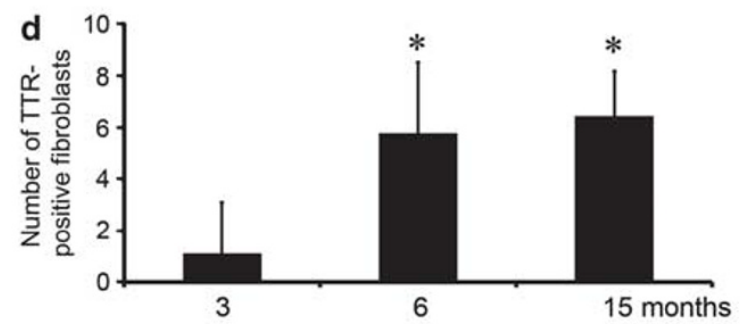

e

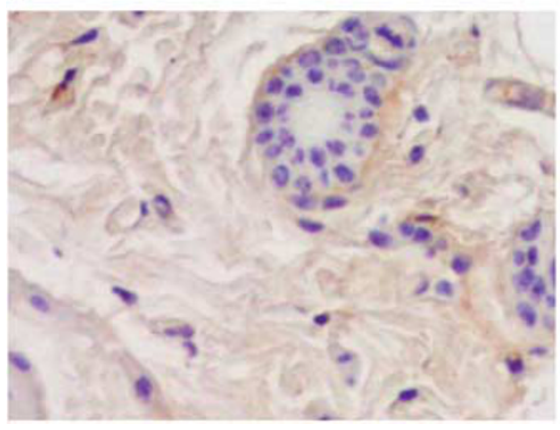

f g

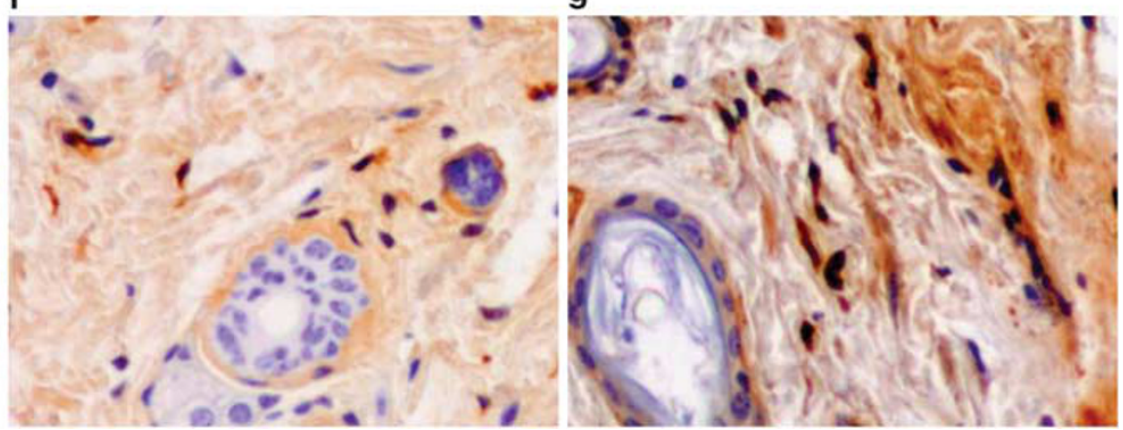

Figure 4 TTR-immunopositive fibroblasts in transgenic mice. (a) TTR immunostaining of a skin sample from transgenic mouse expressing the human amyloidogenic V30M TTR. Insets show boxed areas at greater magnification representing macrophages (upper image) and fibroblasts (lower image). Magnification $\times$ 200. (b) Confocal microscopy image with anti-TTR antibody (green), fibroblast marker (S100A4; red), and DAPI (blue). (c) Confocal microscopic image with anti-TTR antibody (green), macrophage marker (Iba1; red), and DAPI (blue). (d) Semiquantification of the number of TTRimmunopositive fibroblasts in mouse skin of different ages. (e-g) Representative images of TTR immunostaining of skin samples from transgenic mice at the ages of $3(\mathbf{e}), 6(\mathbf{f})$, and $15(\mathbf{g})$ months. Magnification $\times 400 .{ }^{*} P<0.01$.

Also, in the heart, certain fibroblasts from FAP patients manifested TTR immunoreactivity in their cytoplasm, especially fibroblasts in the epicardium and endocardium (Figures 5f-h).

\section{DISCUSSION}

Amyloid deposits preferentially in the ECM, especially at basement membranes, in most types of amyloidosis, including Alzheimer's disease, AL amyloidosis, AA amyloidosis, and TTR-related amyloidosis. ${ }^{22,23}$ The ECM microenvironment is thought to have a key role in amyloid fibril formation in TTR-related amyloidosis, because prefibrillar aggregate and fibrillar forms of TTR exist in the ECM generated from blood-soluble TTR.

Studies on long-term course of FAP patients after liver transplantation showed that the total amount of amyloid deposits in abdominal fat tissues decreased, and contribution of WT TTR to mutant TTR increased, which may indicate the presence of a dynamic process of amyloid fibril formation and metabolism in the ECM. ${ }^{9,10}$

The ECM maintains tissue architecture and homeostasis and contains numerous components, including collagens, proteoglycans, glycosaminoglycans, growth factors, and proteases, that are produced and secreted by various stromal 
a

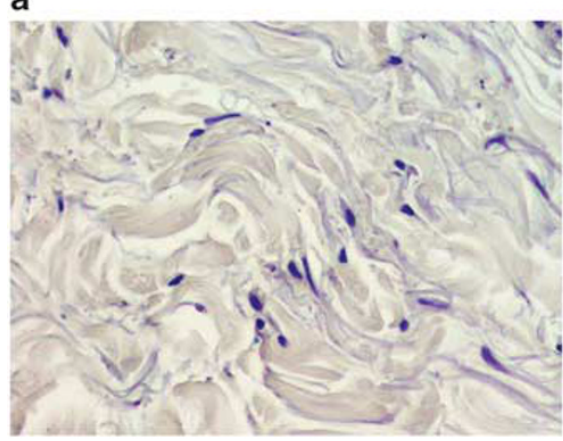

d

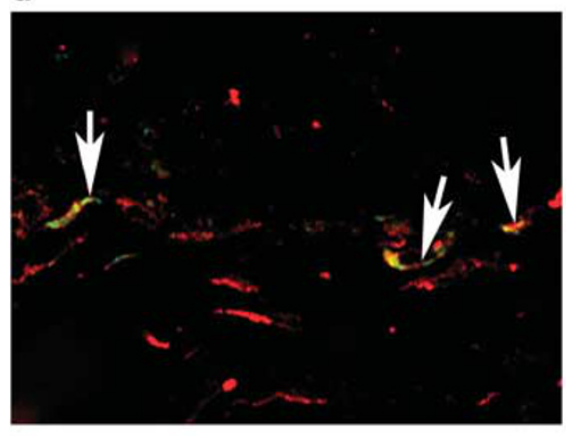

b

$c$

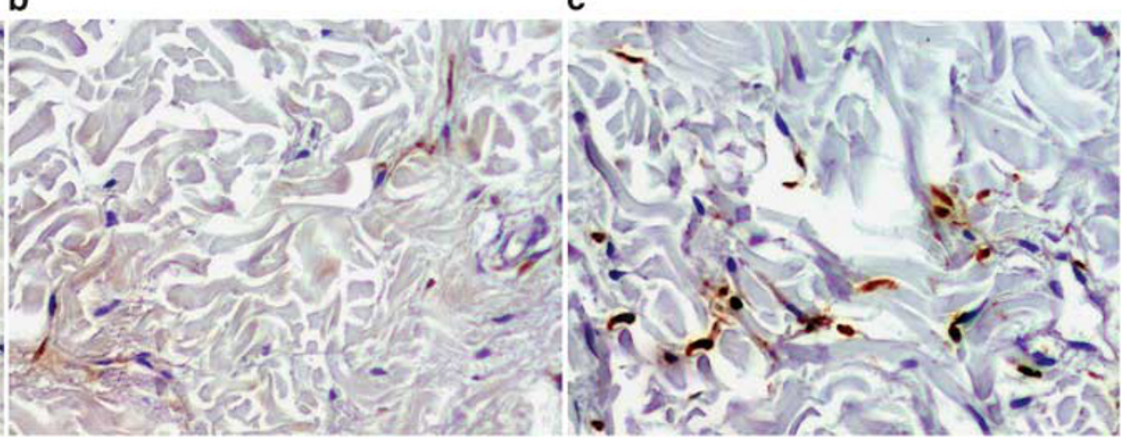

e

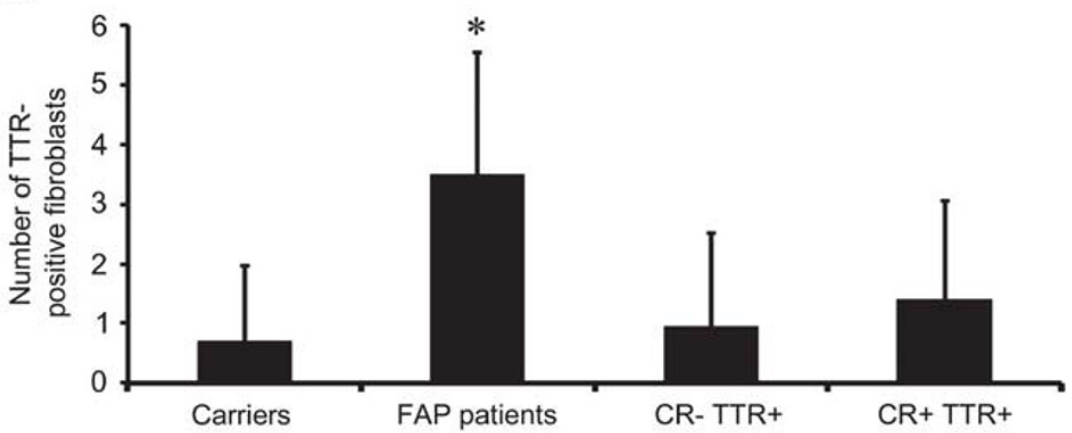

g

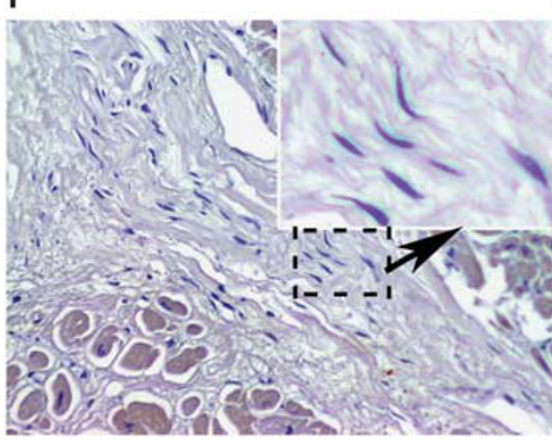

h
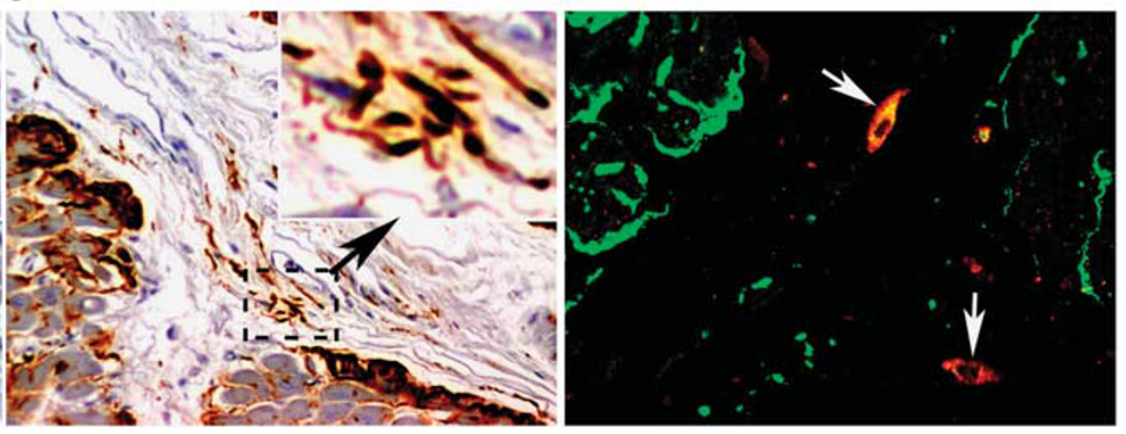

Figure 5 TTR-immunopositive fibroblasts in human tissues. (a-c) Representative images of TTR immunostaining of skin specimens from a (a) control subject, (b) asymptomatic carrier, and (c) FAP patient. Magnification $\times 200$. (d) Confocal microscopy image of a skin specimen from an FAP patient with anti-TTR antibody (green) and fibroblast marker (S100A4; red). Magnification $\times 400$. (e) Semiquantification of the number of TTR-immunopositive fibroblasts in the skin from asymptomatic carriers, FAP patients, and FAP domino liver transplant recipients. ${ }^{*} P<0.05$. (f, $\mathbf{g}$ ) Representative images of TTR immunostaining of heart specimens from (f) control and (g) FAP patients. Magnification $\times 200$. (h) Confocal microscopy image of a heart specimen from an FAP patient with anti-TTR antibody (green) and fibroblast marker (S100A4; red). Magnification $\times 400$. Insets show boxed areas at greater magnification. Arrows point to TTR-immunopositive fibroblasts.

cells, predominantly fibroblasts. ${ }^{24}$ Fibroblasts, together with macrophages, also have a central role in degrading and remodelling the ECM. Although, different types of cells reportedly exhibit varying degrees of TTR internalization activity in vitro, ${ }^{13-15}$ fibroblasts and macrophages have higher migratory potential and more accessibility to TTR deposits in ECM. Previous ultrastructural analyses revealed amyloid deposits at the outer layer of the basement membrane, which occurs at the base of all epithelial linings, underlying endothelia of capillaries and venules, and around Schwann cells, adipocytes, and muscle cells. ${ }^{22,25}$ Therefore, these cells are separated from amyloid deposits by their own basement membranes, and they barely make contact and degrade amyloid fibrils. Fibroblasts and macrophages, however, are in close proximity to amyloid deposits but their interactions are still unclear.

Macrophages perform a significant phagocytic function: removing dead cell materials, necrotic tissue components, and foreign materials such as pathogens, and they also reportedly phagocytosed and degraded amyloid $\beta(\mathrm{A} \beta)$, $\beta_{2}$-microglobulin $\left(\beta_{2} \mathrm{~m}\right)$, and AA amyloid fibrils. ${ }^{26-29}$ Conversely, macrophages also reportedly promoted amyloid formation by phagocytosis of amyloid precursor proteins, including $\mathrm{A} \beta$ and SAA. ${ }^{29,30}$ 
Table 1 Presence of TTR-immunopositive fibroblasts in skin tissues obtained from patients and controls

Domino LT recipients

Controls Carriers FAP patients $\mathrm{CR}-\mathrm{TTR}-\mathrm{CR}-\mathrm{TTR}+\mathrm{CR}+\mathrm{TTR}+$

$\begin{array}{llllll}0 / 4 & 1 / 4 & 3 / 4 & 0 / 5 & 2 / 5 & 4 / 5\end{array}$

Abbreviations: LT, liver transplant; $C R$, Congo red staining; $T R R$, anti-TTR immunostaining.

Fibroblasts produce ECM remodelling enzymes, including matrix metalloproteinases (MMPs) and their specific inhibitors (tissue inhibitor of metalloproteinases). ${ }^{31}$ MMPs work cooperatively to degrade most ECM components. MMPs 2,3 , and 9 were shown to degrade $\mathrm{A} \beta$ fibrils in vitro, ${ }^{32}$ and are upregulated in brain tissues obtained from Alzheimer's disease patients and transgenic model mice. ${ }^{33}$ Similarly, MMPs degraded TTR fibrils and prefibrillar aggregates in vitro and were upregulated in FAP patients, ${ }^{34}$ transgenic animals, ${ }^{35}$ and a cultured cell line. ${ }^{36}$

In this report, we show the capacity of fibroblasts to endocytose and degrade small TTR aggregates in lysosomes, both 'in vitro' and in an 'in vivo' model, after s.c. injection of TTR aggregates into WT mice, whereby soluble and aggregated TTR were endocytosed by fibroblasts and macrophages that efficiently cleared both types of TTRs with time. Recently, intracellular localization of TTR V30M in glomerular podocytes as well as in kidney ECM was shown in transgenic mice. Increased deposition was demonstrated in mice housed in non-specific pathogen-free conditions, suggesting environmental conditions influence both glomerular deposition and podocytic localization of TTRV $30 \mathrm{M} .{ }^{37} \mathrm{Simi}$ larly, a recent study with a mutant TTR transgenic fly model revealed that TTR aggregates were internalized by body fat cells in vivo. ${ }^{38}$ The authors hypothesized that the prefibrillar TTR aggregates matured inside the cells and amyloid fibrils released by cell death. In our cultured cell line and TTRinjected mouse skin, however, formation of amyloid fibrils inside the cytoplasm was not observed.

Intracellular accumulation of aggregated material derived from the ECM was previously reported, not only in fibroblasts but also from other cell types. In one research study of $\beta_{2} \mathrm{~m}$ amyloidosis, in which amyloid fibrils were deposited mainly in the synovium and cartilage, human synovial fibroblasts endocytosed $\beta_{2} \mathrm{~m}$ that had been modified with advanced glycation end products. ${ }^{39}$ Another cell type, astrocytes, which are the most abundant cells in the central nervous system, have functions similar to those of fibroblasts in the maintenance of homeostasis, in which these cells produce ECM components and MMPs. ${ }^{40,41}$ Previous studies provided evidence from brain specimens from Alzheimer's disease patients that astrocytes degraded $\mathrm{A} \beta$ oligomers and amyloid fibrils in vitro and in vivo and manifested intracellular $\mathrm{A} \beta .^{42,43}$ Similarly, in primary cutaneous amyloidosis, intracellular oligomers were observed in the basal cell layer of the epidermis and in surrounding cells, including fibroblasts. ${ }^{44}$

As Figures 4 and 5 illustrate, some fibroblasts in tissues obtained from FAP patients, asymptomatic carriers, domino liver transplant recipients, and transgenic mice contained TTR. It is known that biosynthesis and serum concentrations of TTR decreased with disease progression, but extracellular TTR aggregates accumulated at an accelerated rate. ${ }^{45}$ In our study, abundance of TTR-immunoreactive fibroblasts showed a tendency to increase with progression of amyloid deposition. Most likely, dysfunctional clearance of TTR aggregates by fibroblasts resulting from inability of lysosomes to deal with the intracellular load of aggregated TTR material may cause development and progression of TTR-related amyloidosis. In addition to the intracellular uptake of TTR, as shown here, fibroblasts may have the ability to degrade TTR aggregates and fibrils extracellularly by secreting MMPs. Further studies are needed to clarify these multiple cellular and molecular mechanisms of fibroblasts in the clearance of TTR aggregates, which may provide etiological and therapeutic clues in TTR-related amyloidosis.

Supplementary Information accompanies the paper on the Laboratory Investigation website (http://www.laboratoryinvestigation.org)

\section{ACKNOWLEDGEMENTS}

We would like to thank Paula Gonçalves from IBMC for tissue specimen preparation; Paula Sampaio and Joana Magalhães for support with confocal microscopy and oligomer experiments, respectively. This work is supported by grants from the Amyloidosis Research Committee, the Pathogenesis, Therapy of Hereditary Neuropathy Research Committee, the Surveys and Research on Specific Disease, the Ministry of Health and Welfare of Japan, and Grants-in-Aid for Scientific Research: Strategic Young Researcher Overseas Visits Program for Accelerating Brain Circulation (S2210) from the Ministry of Education, Science, Sports and Culture of Japan to YA and YM and FEDER funds through the Operational Competitiveness ProgrameCOMPETE and by national funding from Portuguese Foundation for Science and Technology (FCT) under the projects FCOMP-01-0124-FEDER-022718 (PEST-C/SAU/LA0002/2011 and PTDC/SAU-ORG/111313/2009) to MJS, and fellowship to NPG (SFRH/BD/74304/2010).

\section{DISCLOSURE/CONFLICT OF INTEREST}

The authors declare no conflict of interest.

1. Ando $Y$, Nakamura $M$, Araki S. Transthyretin-related familial amyloidotic polyneuropathy. Arch Neurol 2005;62:1057-1062.

2. Rapezzi C, Quarta CC, Riva L, et al. Transthyretin-related amyloidoses and the heart: a clinical overview. Nat Rev Cardiol 2010;7:398-408.

3. Hammarström $P$, Jiang $X$, Hurshman $A R$, et al. Sequence-dependent denaturation energetics: a major determinant in amyloid disease diversity. Proc Natl Acad Sci USA 2002;99(Suppl 4):16427-16432.

4. Ihse E, Ybo A, Suhr O, et al. Amyloid fibril composition is related to the phenotype of hereditary transthyretin V30M amyloidosis. J Pathol 2008;216:253-261.

5. Takahashi K, Yi S, Kimura Y, et al. Familial amyloidotic polyneuropathy type 1 in Kumamoto, Japan: a clinicopathologic, histochemical, immunohistochemical, and ultrastructural study. Hum Pathol 1991; 22:519-527.

6. Sousa MM, Cardoso I, Fernandes R, et al. Deposition of transthyretin in early stages of familial amyloidotic polyneuropathy: evidence for toxicity of nonfibrillar aggregates. Am J Pathol 2001;159:1993-2000. 
7. Sousa MM, Ferrão J, Fernandes R, et al. Deposition and passage of transthyretin through the blood-nerve barrier in recipients of familial amyloid polyneuropathy livers. Lab Invest 2004;84:865-873.

8. Santos SD, Fernandes R, Saraiva MJ. The heat shock response modulates transthyretin deposition in the peripheral and autonomic nervous systems. Neurobiol Aging 2010;31:280-289.

9. Tsuchiya A, Yazaki M, Kametani F, et al. Marked regression of abdominal fat amyloid in patients with familial amyloid polyneuropathy during long-term follow-up after liver transplantation. Liver Transpl 2008;14:563-570.

10. Tsuchiya-Suzuki A, Yazaki M, Kametani F, et al. Wild-type transthyretin significantly contributes to the formation of amyloid fibrils in familial amyloid polyneuropathy patients with amyloidogenic transthyretin Val30Met. Hum Pathol 2011:42:236-243.

11. Makover A, Moriwaki $H$, Ramakrishnan $\mathrm{R}$, et al. Plasma transthyretin. Tissue sites of degradation and turnover in the rat. J Biol Chem 1988;263:8598-8603.

12. Longo Alves I, Hays MT, Saraiva MJ. Comparative stability and clearance of [Met30]transthyretin and [Met119]transthyretin. Eur J Biochem 1997;249:662-668.

13. Sousa MM, Saraiva MJ. Internalization of transthyretin. Evidence of a novel yet unidentified receptor-associated protein (RAP)-sensitive receptor. J Biol Chem 2001;276:14420-14425.

14. Sousa MM, Norden AG, Jacobsen C, et al. Evidence for the role of megalin in renal uptake of transthyretin. J Biol Chem 2000;275:38176-38181.

15. Fleming $C E$, Mar FM, Franquinho $F$, et al. Transthyretin internalization by sensory neurons is megalin mediated and necessary for its neuritogenic activity. J Neurosci 2009;29:3220-3232.

16. Macedo B, Batista AR, Ferreira N, et al. Anti-apoptotic treatment reduces transthyretin deposition in a transgenic mouse model of Familial Amyloidotic Polyneuropathy. Biochim Biophys Acta 2008; 1782:517-522.

17. Furuya $H$, Saraiva MJ, Gawinowicz MA, et al. Production of recombinant human transthyretin with biological activities toward the understanding of the molecular basis of familial amyloidotic polyneuropathy (FAP). Biochemistry 1991;30:2415-2421.

18. Teixeira PF, Cerca F, Santos SD, et al. Endoplasmic reticulum stress associated with extracellular aggregates. Evidence from transthyretin deposition in familial amyloid polyneuropathy. J Biol Chem 2006 281:21998-22003.

19. Cardoso I, Almeida MR, Ferreira N, et al. Comparative in vitro and ex vivo activities of selected inhibitors of transthyretin aggregation: relevance in drug design. Biochem J 2007;408:131-138.

20. Bolmont T, Clavaguera $\mathrm{F}$, Meyer-Luehmann $\mathrm{M}$, et al. Induction of tau pathology by intracerebral infusion of amyloid- $\beta$-containing brain extract and by amyloid- $\beta$ deposition in APP $\times$ Tau transgenic mice. Am J Pathol 2007;171:2012-2020.

21. Kohno K, Palha JA, Miyakawa K, et al. Analysis of amyloid deposition in a transgenic mouse model of homozygous familial amyloidotic polyneuropathy. Am J Pathol 1997;150:1497-1508.

22. Misumi $Y$, Ando $Y$, Ueda $M$, et al. Chain reaction of amyloid fibril formation with induction of basement membrane in familial amyloidotic polyneuropathy. J Pathol 2009;219:481-490.

23. Narindrasorasak S, Altman RA, Gonzalez-DeWhitt $\mathrm{P}$, et al. An interaction between basement membrane and Alzheimer amyloid precursor proteins suggests a role in the pathogenesis of Alzheimer's disease. Lab Invest 1995;72:272-282.

24. Rhee S. Fibroblasts in three dimensional matrices: cell migration and matrix remodeling. Exp Mol Med 2009;41:858-865.

25. Yamaguchi $\mathrm{H}$, Yamazaki $\mathrm{T}$, Lemere $\mathrm{CA}$, et al. Beta amyloid is focally deposited within the outer basement membrane in the amyloid angiopathy of Alzheimer's disease. An immunoelectron microscopic study. Am J Pathol 1992;141:249-259.

26. Tolón RM, Núñez $\mathrm{E}$, Pazos MR, et al. The activation of cannabinoid CB2 receptors stimulates in situ and in vitro beta-amyloid removal by human macrophages. Brain Res 2009;1283:148-154.

27. Argilés A, García García M, Mourad G. Phagocytosis of dialysis-related amyloid deposits by macrophages. Nephrol Dial Transplant 2002;17:1136-1138.

28. Nyström SN, Westermark GT. AA-Amyloid is cleared by endogenous immunological mechanisms. Amyloid 2012;19:138-145.

29. Friedrich RP, Tepper K, Rönicke R, et al. Mechanism of amyloid plaque formation suggests an intracellular basis of Abeta pathogenicity. Proc Natl Acad Sci USA 2010;107:1942-1947.

30. Kluve-Beckerman B, Manaloor JJ, Liepnieks JJ. A pulse-chase study tracking the conversion of macrophage-endocytosed serum amyloid A into extracellular amyloid. Arthritis Rheum 2002;46:1905-1913.

31. Biolo A, Ramamurthy S, Connors LH, et al. Matrix metalloproteinases and their tissue inhibitors in cardiac amyloidosis: relationship to structural, functional myocardial changes and to light chain amyloid deposition. Circ Heart Fail 2008;1:249-257.

32. Miners JS, Baig S, Palmer J, et al. Abeta-degrading enzymes in Alzheimer's disease. Brain Pathol 2008;18:240-252.

33. Backstrom JR, Miller CA, Tökés ZA. Characterization of neutral proteinases from Alzheimer-affected and control brain specimens: identification of calcium-dependent metalloproteinases from the hippocampus. J Neurochem 1992;58:983-992.

34. Sousa MM, do Amaral JB, Guimarães $A$, et al. Up-regulation of the extracellular matrix remodeling genes, biglycan, neutrophil gelatinaseassociated lipocalin, and matrix metalloproteinase-9 in familial amyloid polyneuropathy. FASEB J 2005;19:124-126.

35. Cardoso I, Saraiva MJ. Doxycycline disrupts transthyretin amyloid: evidence from studies in a FAP transgenic mice model. FASEB J 2006;20:234-239.

36. Cardoso I, Brito M, Saraiva MJ. Extracellular matrix markers for disease progression and follow-up of therapies in familial amyloid polyneuropathy V30M TTR-related. Dis Markers 2008;25:37-47.

37. Petrakis I, Mavroeidi V, Stylianou K, et al. Human TTRV30M localization within podocytes in a transgenic mouse model of transthyretin related amyloidosis: does the environment play a role? Transgenic Res 2013;22:101-116.

38. Pokrzywa M, Dacklin I, Vestling $M$, et al. Uptake of aggregating transthyretin by fat body in a Drosophila model for TTR-associated amyloidosis. PLoS One 2010;5:e14343.

39. O'Neill KD, Chen NX, Wang $M$, et al. Cellular uptake of beta2M and AGE-beta2M in synovial fibroblasts and macrophages. Nephrol Dial Transplant 2003;18:46-53.

40. Li W, Poteet $E$, Xie L, et al. Regulation of matrix metalloproteinase 2 by oligomeric amyloid $\beta$ protein. Brain Res 2011;1387:141-148.

41. Wiese S, Karus M, Faissner A. Astrocytes as a source for extracellular matrix molecules and cytokines. Front Pharmacol 2012;3:120.

42. Wyss-Coray T, Loike JD, Brionne TC, et al. Adult mouse astrocytes degrade amyloid-beta in vitro and in situ. Nat Med 2003;9:453-457.

43. Lasagna-Reeves CA, Kayed R. Astrocytes contain amyloid- $\beta$ annular protofibrils in Alzheimer's disease brains. FEBS Lett 2011;585: 3052-3057.

44. Clos AL, Lasagna-Reeves CA, Kelly B, et al. Role of oligomers in the amyloidogenesis of primary cutaneous amyloidosis. J Am Acad Dermatol 2011;65:1023-1031.

45. Sekijima Y, Tokuda T, Kametani F, et al. Serum transthyretin monome in patients with familial amyloid polyneuropathy. Amyloid 2001;8: 257-262. 